comparisons - and therefore has universal appeal. This will no doubt contribute to the increasing consistency (some might say uniformity) of psychiatric practice worldwide. This book is perfect fodder in preparing for membership examinations it is comprehensive and relevant, while at the same time being accessible and fluent.

It would appear that, for the time being at least, the textbook is alive and well. In the current three-horse race the clear winner is the Shorter Oxford Textbook, with the Introductory Textbook a close second.

Gelder, M. G., López-lbor Jr, J. J. \& Andreasen, N. (eds) (2000) New Oxford Textbook of Psychiatry. Oxford: Oxford University Press.

Ronan Mclvor Consultant Psychiatrist, Maudsley Hospital, Denmark Hill, London SE5 8AZ, UK

\section{Obsessive Compulsive Disorder: A Practical Guide}

Edited by Naomi Fineberg, Donatella Marazziti \& Dan J. Stein. London: Martin Dunitz. 2001. 228 pp. £24.95 (hb). ISBN I 85317919 ।

In the past few years, the explosion of interest in the aetiology and treatment of obsessive-compulsive disorder (OCD) has led to the publication of several multiauthor books on the subject. They all attempt to summarise and integrate an extensive research literature and clinical practice experience. This new book achieves this goal well and really does present a practical approach to evaluation and treatment of the patient with OCD, as well as embedding this knowledge within an authoritative summary of current academic thinking. It perhaps lacks the clinical warmth and immediacy of Koran's book (1999) (although he has a good chapter on quality-of-life issues in this volume), nor does it have the comprehensive research reviews of the 1999 volume edited by Goodman et al (Goodman also has a helpful chapter in this book on assessment instruments). Swinson et al's excellent 1998 book now has a 2002 second edition, and the volume edited by Maj et al in 2000 is presented in an unusual style, with chapters and linked commentaries. All of these books cover much of the same ground, but for brevity and practicality, Fineberg and colleagues could well be the best choice.
In its 180 or so pages of text (with an additional appendix of useful questionnaires, contacts, etc.), it can be read in one sitting by a clinician wanting to update or to begin work in a new specialist area. Chapters on the biology and pharmacology of OCD take up about half of the book, which realistically reflects the emphasis that there has been on these aspects in current research. Similarly, the most robust evidence for treatment responsiveness is with medication, and the book correctly emphasises how for many patients this remains the mainstay of treatment. There are practical chapters on the use of medication, including the treatment of refractory OCD.

Psychological theory and treatments are not neglected, and although there continues to be sparse clinical trial data on the relative or combined effects of psychological and pharmacological treatment, the case is clearly made for the benefits of cognitive-behavioural therapy. A wide-ranging chapter on the psychotherapies in OCD is not constrained by any particular theoretical framework, but reviews the clinical evidence for efficacy. This is not to dismiss the importance of theory for furthering the field and developing new treatments, and a final chapter on integrated approaches attempts to reconcile the evidence on subcortical/cortical brain dysfunction in OCD with psychological theories of maladaptive risk appraisal and excessive sense of responsibility. Once again, this is done in a 'practical' way, reflecting on the

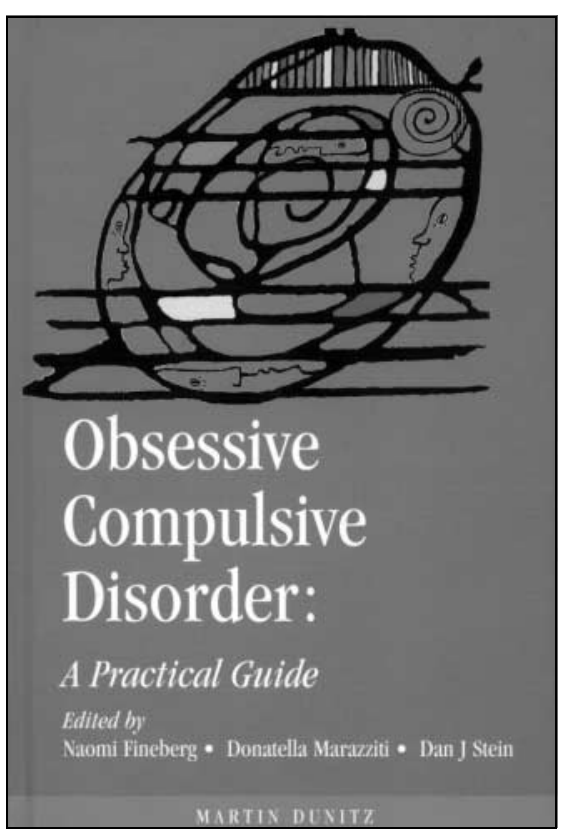

importance in therapy of an explanatory model with which the patient can engage and experiment.

Although short, this book does not succumb to over-simplified views of OCD. For example, it acknowledges that OCD is likely to be a heterogeneous group of disorders rather than a single disease and that a simplistic 'serotonin-deficit' hypothesis is not likely to be adequately explanatory. This broadness of approach leads the reader through the challenges of treatment, including working with young people and resistant cases. The overall theme of integrating a 'brain-mind' approach in the understanding and treatment of OCD is successfully achieved. For the critical and practical clinician or researcher, wanting to incorporate biological and psychological theories into their thinking and practice in OCD, this book represents an excellent foundation.

Goodman, W., Rudorfer, M. V. \& Maser, J. D. (eds) (1999) Obsessive-Compulsive Disorder. Rahwah, N: Lawrence Erlbaum.

Koran, L. M. (1999) Obsessive-Compulsive and Related Disorders in Adults. Cambridge: Cambridge University Press.

Maj, M. (ed.) (2000) Obsessive-Compulsive Disorder. Chichester: John Wiley \& Sons.

Swinson, R. P., Antony, M. M., Rachman, S., et a (eds) (2002) Obsessive-Compulsive Disorder. New York: Guilford Press.

I. Heyman Michael Rutter Centre for Children and Young People, Obsessive-Compulsive Disorder Clinic, Maudsley Hospital, De Crespigny Park, London SE5 8AZ, UK

\section{Mental Health, Race and Culture (2nd edn)}

By Suman Fernando. London: Palgrave. 2002. 256 pp. $€ 16.99$ (pb). ISBN 0333960262

The first edition of Suman Fernando's book in 1991 was perhaps the most influential of the 'second wave' of British transcultural psychiatry. Not especially original in sources nor themes, it was nevertheless an outstanding critique of psychiatric practice in the area of ethnicity and race. It resolutely commented on the failures of our profession to provide equal and appropriate treatment for minority ethnic groups, and sustained a vigorous attack on psychiatric diagnoses as presumed entities rather than explanatory models, their being 